\title{
Online Social Network Emergency Public Event Information Propagation and Nonlinear Mathematical Modeling
}

\author{
Xiaoyang Liu, ${ }^{1,2}$ Chao Liu, ${ }^{2}$ and Xiaoping Zeng' \\ ${ }^{1}$ Postdoctoral Research Station of Information and Communication Engineering, Chongqing University, Chongqing 400030, China \\ ${ }^{2}$ School of Computer Science and Engineering, Chongqing University of Technology, Chongqing 400054, China \\ Correspondence should be addressed to Xiaoyang Liu; lxy3103@163.com
}

Received 23 March 2017; Revised 26 May 2017; Accepted 31 May 2017; Published 28 June 2017

Academic Editor: Junhu Ruan

Copyright (c) 2017 Xiaoyang Liu et al. This is an open access article distributed under the Creative Commons Attribution License, which permits unrestricted use, distribution, and reproduction in any medium, provided the original work is properly cited.

\begin{abstract}
Emergency public event arises everyday on social network. The information propagation of emergency public event (favorable and harmful) is researched. The dynamics of a susceptible-infected-susceptible and susceptible-infected-removed epidemic models incorporated with information propagation of emergency public event are studied. In particular, we investigate the propagation model and the infection spreading pattern using nonlinear dynamic method and results obtained through extensive numerical simulations. We further generalize the model for any arbitrary number of infective network nodes to mimic existing scenarios in online social network. The simulation results reveal that the inclusion of multiple infective node achieved stability and equilibrium in the proposed information propagation model.
\end{abstract}

\section{Introduction}

1.1. Background and Status. Emergency public events happen every day, such that the bitcoin virus is spreading all over the world in May 2017. In today's world, individuals interact with each other in more complicated patterns than ever. Some individuals engage through complex networks, while some communicate only through conventional ways. So, it is easy to cause infectious diseases. Therefore, it is very necessary for us to understand the dynamics of epidemic propagation characteristic and disease dynamic behavior. Epidemiology is the study of disease distribution rule and the influence of the epidemic dynamic model. The critical epidemic thresholds and the infection spreading pattern using meanfield approximation (MFA) and results obtained through extensive numerical simulations are researched in $[1,2]$. A new susceptible-infected-susceptible (SIS) epidemic model incorporated with multistage infection (infection delay) and an infective medium (propagation vector) over complex networks [3-6]. In [7-9], a novel ISIR epidemic model with nonlinear forces of infection to characterize the epidemic spread on social contact networks with the consideration of the "crowding" or "protection effect" is proposed. For this class of dynamic networks, [10-14] derive an epidemic threshold, considering the susceptible-infected-susceptible epidemic model. First, an epidemic probabilistic model is developed assuming independence between states of nodes. Then they identify the conditions under which the epidemic dies out by linearizing the underlying dynamical system and analyzing its asymptotic stability around the origin. The concept of joint spectral radius is then used to derive the epidemic threshold, which is later validated using several networks. A simplified version of the epidemic threshold is proposed for undirected networks. References $[8,9,15]$ investigated the mathematical epidemic model, SEIR (SusceptibleExposed-Infected-Removed), through extensive simulations of the effects of social network on epidemic spread in a Small World (SW) network, to understand how an influenza epidemic spreads through a human population. A combined SEIR-SW model was built, to help understand the dynamics of infectious disease in a community and to identify the main characteristics of epidemic transmission and its evolution over time. The model was also used to examine social network effects to better understand the topological structure of social contact and the impact of its properties. An improved susceptible-infected-susceptible (SIS) epidemic 
spreading model is proposed in order to provide a theoretical method to analyze and predict the spreading of diseases [1620]. This model is based on the following idea: in social networks, the contact probability between nodes is decided by their social distances and their active degrees.

Although classic disease spread dynamic models make some modest success in predicting some particular disease behaviors, they are usually overly simplistic and neglect certain important aspects, like multiple stages, multiple groups, the number of contacts, and other disease states. The rest of this paper is organized as follows. In Section 2, characteristic of information propagation is studied. In Section 3, the information propagation model is prosed. In Section 4, stability and equilibrium of information propagation model are researched, In Section 5, simulation analytical results are presented. Finally, conclusions are drawn in Section 6.

1.2. Motivations. The above discussion implies that novel inequality methods allow us to obtain more emergency public event information propagation model and algorithm. Most references do not consider the dynamic behaviors of emergency public event information propagation by using computer virus propagation theory under online social network. Social network information modeling methods and algorithms are inequality presented in some references. Under the method efficiency, a novel emergency public event information propagation information propagation algorithm and mathematics model could be proposed.

1.3. Our Work and Contributions. In this paper, the emergency public event information propagation modeling problem based on online social network by nonlinear dynamic method is further investigated. By giving more precise estimation for emergency public event information propagation, a new emergency public event information propagation method is obtained. Under this method, some novel results are presented. Based on computer virus propagation theory, the nonlinear mathematics model of emergency public event information propagation is derived. Simulation is employed to indicate the effectiveness of the proposed method and model. The main contributions of this paper are listed as follows. (1) The information propagation process of emergency public event is considered as a process of network virus transmission. The computer virus propagation theory is introduced to the emergency public event information propagation. (2) An emergency public event information propagation method and a new information process model are proposed. (3) A new information propagation structure in online social network is built up. (4) The better performance is obtained by the theoretical simulation results. These results are practical and objective.

\section{Characteristic of Information Propagation}

2.1. Public Event Information Characteristic. Nowadays, the time and speed of the Internet are very huge for emergency

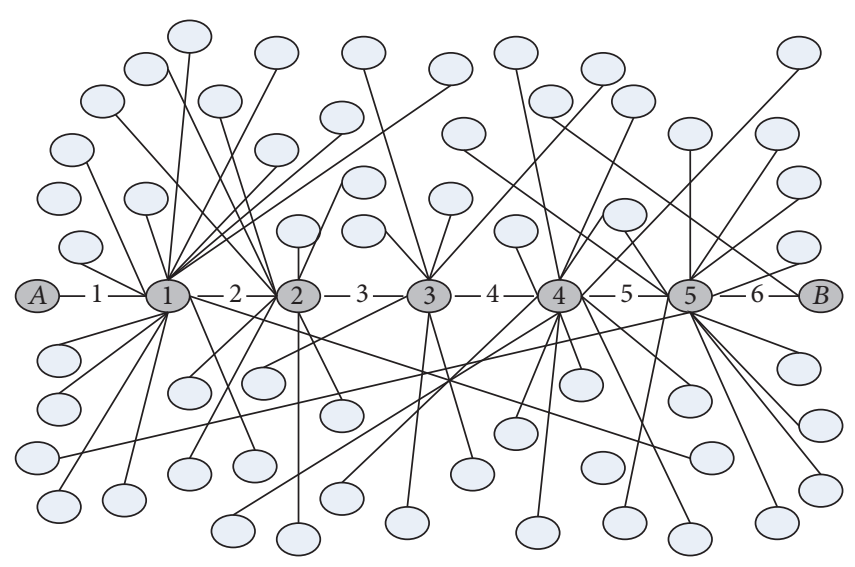

FIGURE 1: The six-degree theory of partition theory.

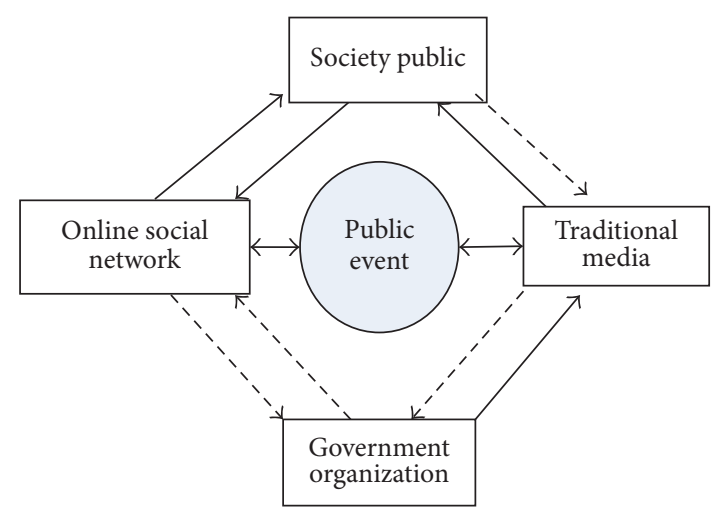

FIGURE 2: The propagation model of public event.

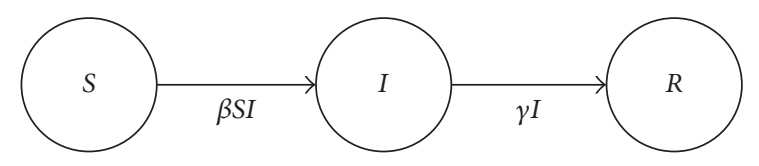

FIGURE 3: The chart model of SIR.

public event information. Emergency public event information is spread exponentially based on six-degree theory of partition theory, which can be seen from Figure 1 .

The propagation model of public event information is shown in Figure 2.

2.2. Traditional Virus and Epidemic Model. The "compartment" model is an essential mathematical model for a long time. The basic idea of the "compartment" model was built by Kermack and Mckendrick. It has received various high praise right from the start.

2.2.1. SIR Model. SIR model [21-26] is divided into three classes, which are susceptible $(S)$, infections $(R)$, and removed $(I)$; their quantity is $S(t), I(t)$, and $R(t)$, respectively. The chart model of SIR is shown in Figure 3. 


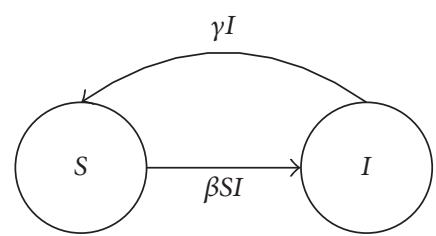

FIgURE 4: The chart model of SIS.

$\beta$ and $\gamma$ are the proportionality coefficients. On the basis of the change rate of the quantity in every compartment, the material balance equation can be obtained:

$$
\begin{aligned}
\frac{\mathrm{d} S}{\mathrm{~d} t} & =-\beta S I, \\
\frac{\mathrm{d} I}{\mathrm{~d} t} & =\beta S I-\gamma I, \\
\frac{\mathrm{d} R}{\mathrm{~d} t} & =\gamma I .
\end{aligned}
$$

2.2.2. SIS Model. Generally speaking, influenza, measles, and other epidemics are spread by viral disease. Immunization is also a key strategy to ensure global health security and for responding to the threat of emerging infections such as pandemic influenza. These diseases are immune to provirus. The model of SIR is suitable for these epidemics. It is likely that most of epidemics do not have immunity to provirus. Then the SIS model [27-30] is proposed by Kermack and Mckendrick. The chart model of SIR is drawn in Figure 4.

The material balance equation can be denoted as

$$
\begin{aligned}
& \frac{\mathrm{d} S}{\mathrm{~d} t}=-\beta S I+\gamma I, \\
& \frac{\mathrm{d} I}{\mathrm{~d} t}=\beta S I-\gamma I .
\end{aligned}
$$

SIS model can reduce the probability of infection and increase the cure rate.

2.2.3. Epidemic Dynamic Model with Time-Lag. The basic fact for the epidemic dynamic mathematics model of time-lag is that the movement rule of a certain moment $t$ depending on current $t$ not only is related to its previous stat $t$ but also is a reflection of certain factors on previous stat $t$. KermackMckendrick model [31-33] is expressed as

$$
\begin{aligned}
& \frac{\mathrm{d} S}{\mathrm{~d} t}=-\beta S I+\gamma I, \\
& \frac{\mathrm{d} I}{\mathrm{~d} t}=\beta S I-\gamma I,
\end{aligned}
$$

where $1 / \gamma$ denotes the average recovery period; $t$ is the time; $S$ is the susceptible; $I$ expresses the infection; $\beta$ is the proportionality coefficient.

\section{Information Propagation Model}

We assume that the total number of network nodes is continuously changed by time. $S(t)$ denotes the susceptible node number which is not infected at time $t . I(t)$ is the infected node number, which has been infected with the virus infections at time $t . R(t)$ expresses the immune node number of susceptible which has immunity to the virus at time $t$. The virus propagation model is shown in Figure 5. $n$ indicates the node number of new added infected nodes. $\beta$ shows the coefficient which reflects the relation between susceptible nodes and infected node. $1-p$ signifies the probability that it is the number of new nodes that is priorly equipped with a firewall directly into immune state. $\mu_{1}, \mu_{2}$, and $\mu_{3}$ express removing probability of the network node three states, respectively. $k$ represents received immunized antivirus measures rate which is the susceptible nodes through effective antivirus software installation in advance measures and a series of antivirus, firewall, and so on. $\tau$ denotes incubation period of the virus. Thus individual susceptibility node infection rate that was infected with the virus can be expressed as $\beta I(t-\tau)$.

Based on the above assumptions and propagation dynamics theory, network time-delay model can be built up as follows:

$$
\begin{aligned}
& \frac{\mathrm{d} S}{\mathrm{~d} t}=p n-\beta S(t) I(T-\tau)-\left(\mu_{1}+k\right) S(t), \\
& \frac{\mathrm{d} I}{\mathrm{~d} t}=\beta S(t) I(T-\tau)-\left(\mu_{2}+k\right) I(t), \\
& \frac{\mathrm{d} R}{\mathrm{~d} t}=(1-p) n+\gamma I(t)-\mu_{3} R(t)+k S(t) .
\end{aligned}
$$

Emergency public event information propagation can be divided into favorable information and harmful information. As for favorable information, $p_{\tau}$ can be expressed as follows:

$$
p_{\tau}= \begin{cases}1-\frac{1-p^{2}}{4 p} \ln \frac{1+p}{1-p}, & \tau=1 \\ \frac{1-p^{2}}{4 p}\left[\left(\frac{1+p}{2}\right)^{\tau-1}-\left(\frac{1-p}{2}\right)^{\tau-1}\right] \frac{1}{\tau-1} \exp \left(\frac{\tau}{30 n}\right), & \tau>1 .\end{cases}
$$


As for harmful information, the $p_{\tau}$ can be expressed as

$$
p_{\tau}= \begin{cases}1-\frac{1-p^{2}}{4 p} \ln \frac{1+p}{1-p}, & \tau=1 \\ \frac{\left(\left(1-p^{2}\right) / 4 p\right)\left[((1+p) / 2)^{\tau-1}-((1-p) / 2)^{\tau-1}\right](1 /(\tau-1))}{\exp (\tau / 25 n)}, & \tau>1 .\end{cases}
$$

\section{Stability Analysis}

Theorem 1 (see [33-35]). When $R_{0} \leq 1$, the local no propagation equilibrium point $P$ is becoming asymptotic stability. When $R_{0}>1$, no propagation equilibrium point $P$ is instability.

Proof. Jacobi matrix of $P$ can be expressed as follows:

$$
\left[\begin{array}{ccc}
\alpha-2 \alpha H-(\beta+\varepsilon) & 0 & 0 \\
\beta & -\gamma & 0 \\
\varepsilon & \gamma & -\eta
\end{array}\right]
$$

The eigenvalue can be denoted as

$$
\begin{aligned}
& \lambda_{1}=\alpha-2 \alpha H-(\beta+\varepsilon), \\
& \lambda_{2}=-\gamma, \\
& \lambda_{3}=-\mu .
\end{aligned}
$$

As for $P, H=0$. So $\lambda_{1}=\alpha-(\beta+\varepsilon)$. As can be known for the setting of parameters, $\lambda_{2}$ and $\lambda_{3}$ are the negative real number. So, when $R_{0}<1, \lambda_{1}<0$; when $R_{0}>1, \lambda_{1}>0$.

\section{Simulation Analysis}

The network dynamics epidemic propagation process can be simulated by using continuous time algorithm. Each node in the network at any time $t$ can be denoted by $S(t), I(t)$, and $R(t)$. When $t=0$, we can select a node randomly in a network and make their status $I(t)$, and the rest of the nodes are denoted by $S(t)$.

After that, calculate the positive equilibrium point $E_{0}$ of formula (4). When $\tau=1$, the system is asymptotically stable, as can be seen from Figure 6. In the case of other parameters being constant, take different $\tau$ value. For each state node number along with the change of time for numerical simulation, we found that although the delay does not affect the ultimate stability of equilibria, the pace of influence model tends towards equilibrium. When the value $\tau$ is small, formula (4) tends towards equilibrium solution in a short time. When the value $\tau$ is large, system solution to balance time increases. The ubiquity of computer network virus shows what can happen when the technology of virus detection gets distributed. You will be able to realize effective control of the spread of the virus in a short period of time. This is the case of early detection, early antivirus. This aspect shows the virus detection technology in the control of the computer network and the importance of the spread of the virus. On the other hand, it proves the feasibility of this model.

As for the favorable event (Figure 7), when $p$ is ranging from 0.99 to 0.999 , when the waiting time is the initial value, no matter what the value of $p$ is, the occurring probability of emergency public event is 1 . As the wait time is increasing, the value of $p$ is becoming smaller. The curve of waiting time is going down faster. In Figure 7 , red curve represents the probability curve of the initial waiting time; the black curve expresses the improved method's probability curve of the waiting time.

As for the harmful event (Figure 8), when $p$ is ranging from 0.99 to 0.999 , when the waiting time is the initial value, no matter what the value of $p$ is, the occurring probability of emergency public event is 1 . As the wait time is increasing, the value of $p$ is becoming smaller. The curve of waiting time is going down faster.

The predictions of a scale-free network model can be demonstrated in Figure 9.

As can be seen from Figure 9, it is very clear that the model describes the peak time of arrival. The values of the experiment and the calculation of ground surface roughness are accordant basically and also have the same change tendency.

\section{Conclusions}

In this paper, we have formulated an improved mathematical model with information propagation of emergency public event using nonlinear dynamic method. Although a few models were reported in this respect, they were specially focused on the presence of either one of the two factors and did not account for their joint impact on the spreading process. In reality, the proposed model applies to the transmission of diseases in network systems.

The analogy in technological networks includes information propagation of emergency public event via e-mails and file sharing. Through numerical simulations, we demonstrated that the inclusion of delayed infection and infective network node remarkably lowered the traditional method. We also increased the number of network nodes to a considerable time. Such results simply imply that effective and feasible preventive and containment countermeasures are required to lessen the impact of either or both factors to avoid the enhancement and prevalence of infection in networks. A number of modifications to the presented model can be made to add more realism. Though this work targets 


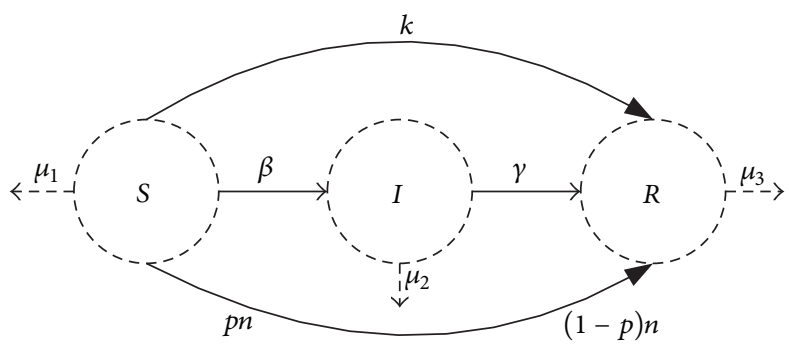

FigURE 5: Virus propagation model.

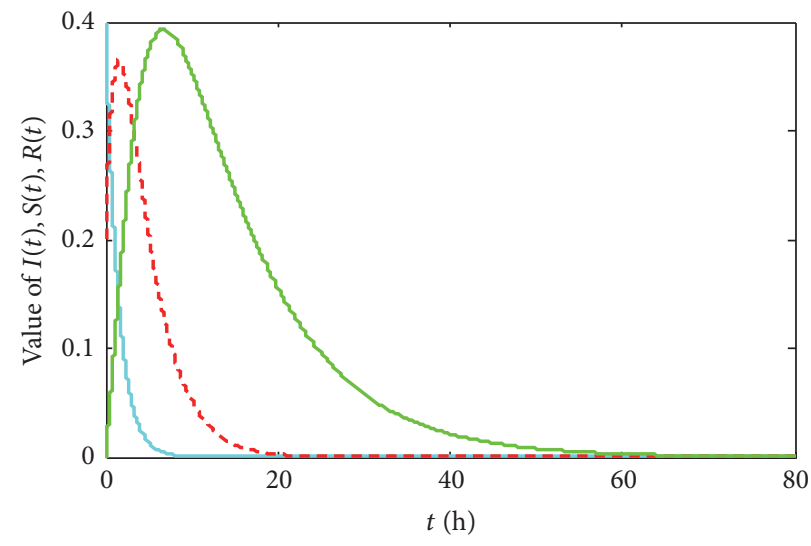

- $I(t)$

$---S(t)$

- $R(t)$

FIGURE 6: System stability simulation of system.
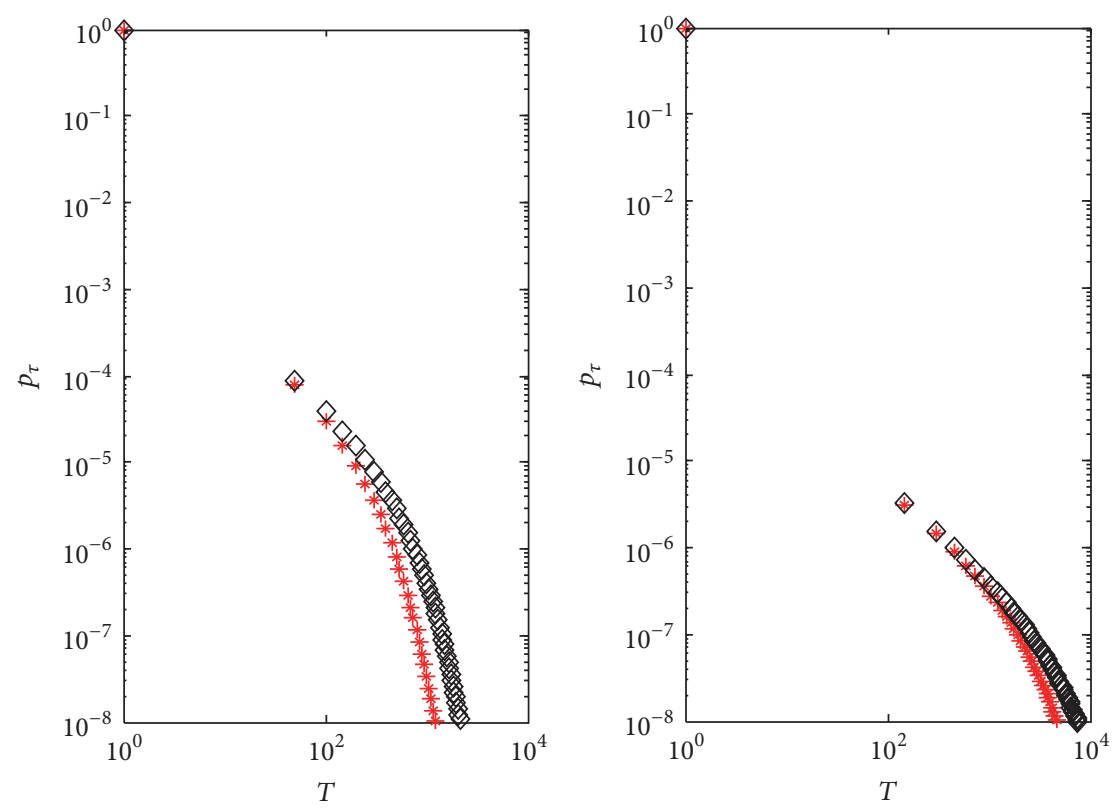

$$
\begin{aligned}
& * \text { Before }(p=0.99) \\
& \diamond \operatorname{After}(p=0.99)
\end{aligned}
$$$$
\text { * Before }(p=0.999)
$$$$
\diamond \operatorname{After}(p=0.999)
$$

FIGURE 7: Favorable event waiting time probability distribution comparison chart $(p=0.99 / 0.999)$. 

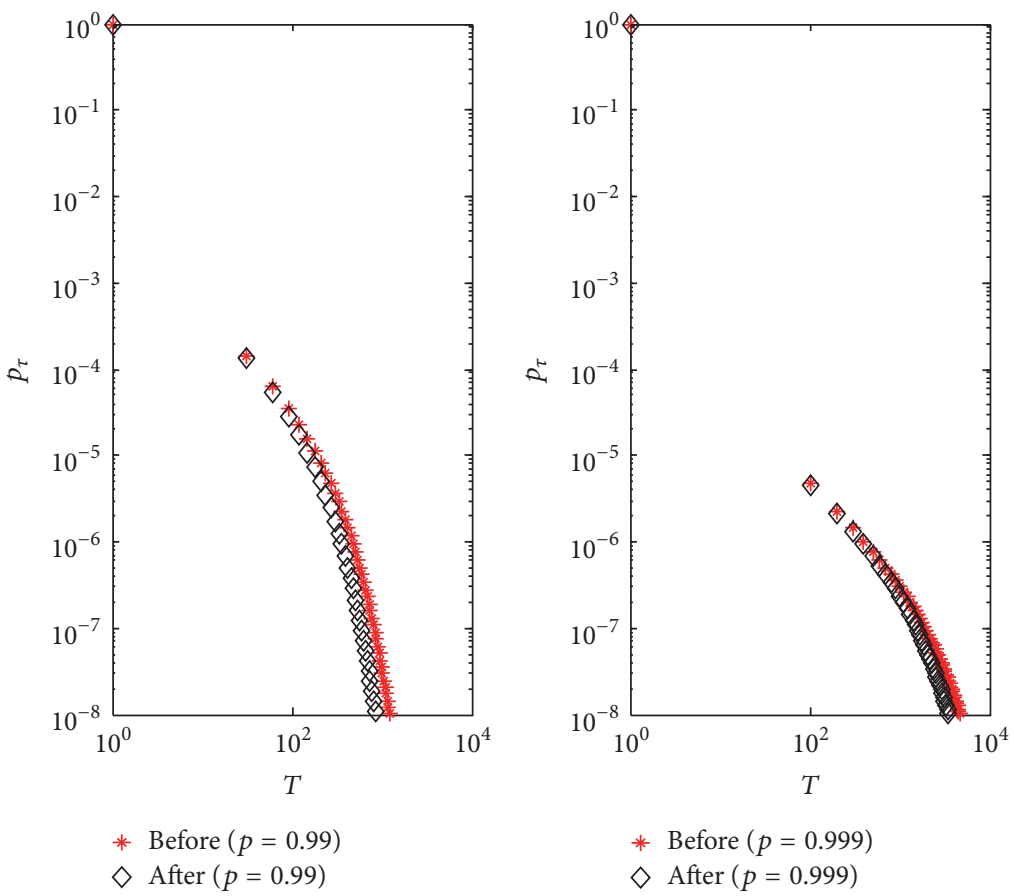

FIGURE 8: Harmful event waiting time probability distribution comparison chart $(p=0.99 / 0.999)$.

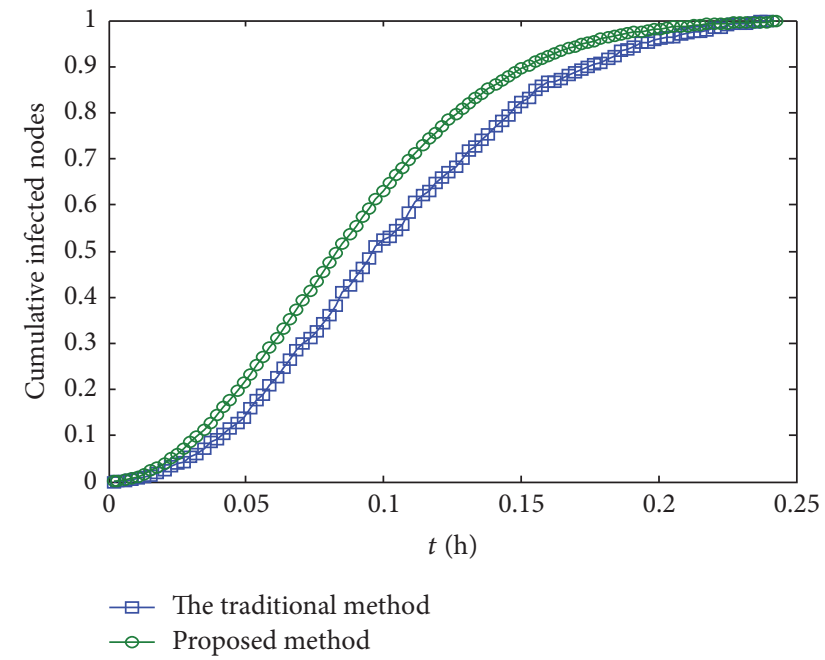

Figure 9: Cumulative infected nodes and time.

the spread of information propagation of emergency public event, the methods presented here could be applied for other applications such as the spread of rumors or ideas through social contact networks.

In the future, we intend to study the impact of infection delay and multiple epidemic propagation vectors on the spreading process in more recent complex models with multiple network layers.

\section{Conflicts of Interest}

The authors declare that there are no conflicts of interest regarding the publication of this paper.

\section{Acknowledgments}

The paper was supported by Young Fund Project of Humanities and Social Sciences Research of Ministry of Education of China (Grant no. 16YJC860010), Chongqing Social Sciences Planning Doctoral Project (2015BS059), Science and Technology Research Program of Chongqing Municipal Education Commission (Grants nos. KJ1600923 and KJ1709206), Natural Science Foundation of China (61571069, 61501065, 61502064, 61503052, and 91438104), and Chongqing Postdoctoral Researchers Research Project Special Funded (Xm2015029).

\section{References}

[1] Q. Wu, S. Chen, and L. Zha, "Epidemic spreading over quenched networks with local behavioral response," Chaos, Solitons \& Fractals, vol. 96, no. 3, pp. 17-22, 2017.

[2] C. Li, X. Yu, W. Yu, G. Chen, and J. Wang, "Efficient Computation for Sparse Load Shifting in Demand Side Management," IEEE Transactions on Smart Grid, vol. 8, no. 1, pp. 250-261, 2016.

[3] H. A. M. Malik, A. W. Mahesar, F. Abid, A. Waqas, and M. R. Wahiddin, "Two-mode network modeling and analysis of dengue epidemic behavior in Gombak, Malaysia," Applied Mathematical Modelling, vol. 43, no. 3, pp. 207-220, 2017.

[4] C. Li, X. Yu, W. Yu, T. Huang, and Z.-W. Liu, "Distributed eventtriggered scheme for economic dispatch in smart grids," IEEE Transactions on Industrial Informatics, vol. 12, no. 5, pp. 17751785, 2016.

[5] G. Chowell, L. Sattenspiel, S. Bansal, and C. Viboud, "Mathematical models to characterize early epidemic growth: A review," Physics of Life Reviews, vol. 18, pp. 66-97, 2016.

[6] S. Mishra, M.-C. Boily, S. Schwartz et al., "Data and methods to characterize the role of sex work and to inform sex work 
programs in generalized HIV epidemics: evidence to challenge assumptions," Annals of Epidemiology, vol. 26, no. 8, pp. 557569, 2016.

[7] Y. Maeno, "Detecting a trend change in cross-border epidemic transmission," Physica A. Statistical Mechanics and its Applications, vol. 457, pp. 73-81, 2016.

[8] C. Li, W. Yu, and T. Huang, "Impulsive synchronization schemes of stochastic complex networks with switching topology: average time approach," Neural Networks, vol. 54, pp. 85-94, 2014.

[9] F. Xiong, Y. Liu, and J. Cheng, "Modeling and predicting opinion formation with trust propagation in online social networks," Communications in Nonlinear Science and Numerical Simulation, vol. 44, pp. 513-524, 2017.

[10] J. Yan, M. Gong, L. Ma, S. Wang, and B. Shen, "Structure optimization based on memetic algorithm for adjusting epidemic threshold on complex networks," Applied Soft Computing Journal, vol. 49, pp. 224-237, 2016.

[11] G. Zhu, G. Chen, and X. Fu, "Effects of active links on epidemic transmission over social networks," Physica A. Statistical Mechanics and its Applications, vol. 468, pp. 614-621, 2017.

[12] Q. Wu and F. Zhang, "Threshold conditions for SIS epidemic models on edge-weighted networks," Physica A. Statistical Mechanics and its Applications, vol. 453, pp. 77-83, 2016.

[13] A. Socievole, F. De Rango, C. Scoglio, and P. Van Mieghem, "Assessing network robustness under SIS epidemics: The relationship between epidemic threshold and viral conductance," Computer Networks, vol. 103, pp. 196-206, 2016.

[14] T. Zhang, L.-X. Yang, X. Yang, Y. Wu, and Y. Y. Tang, "Dynamic malware containment under an epidemic model with alert," Physica A. Statistical Mechanics and its Applications, vol. 470, pp. 249-260, 2017.

[15] L. Wang, M. Sun, S. Chen, and X. Fu, "Epidemic spreading on one-way-coupled networks," Physica A. Statistical Mechanics and its Applications, vol. 457, pp. 280-288, 2016.

[16] C. Li, X. Yu, Z.-W. Liu, and T. Huang, "Asynchronous impulsive containment control in switched multi-agent systems," Information Sciences, vol. 370, pp. 667-679, 2016.

[17] J.-Q. Kan and H.-F. Zhang, "Effects of awareness diffusion and self-initiated awareness behavior on epidemic spreading-an approach based on multiplex networks," Communications in Nonlinear Science and Numerical Simulation, vol. 44, pp. 193203, 2017.

[18] W. Liu, C. Liu, and X. Liu, "A discrete dynamic model for computer worm propagation," in Proceedings of the Mathematics \& Statistics, M. Bohner, Y. Ding, and O. Došlý, Eds., vol. 150, pp. 119-131, Springer, Cham, Switzerland, 2015.

[19] A. Cherif, J. Dyson, P. K. Maini, and S. Gupta, "An agestructured multi-strain epidemic model for antigenically diverse infectious diseases: a multi-locus framework," Nonlinear Analysis. Real World Applications. An International Multidisciplinary Journal, vol. 34, pp. 275-315, 2017.

[20] D. Wanduku, "Complete global analysis of a two-scale network SIRS epidemic dynamic model with distributed delay and random perturbations," Applied Mathematics and Computation, vol. 294, pp. 49-76, 2017.

[21] H. F. Lee, J. Fei, C. Y. S. Chan, Q. Pei, X. Jia, and R. P. H. Yue, "Climate change and epidemics in Chinese history: A multiscalar analysis," Social Science \& Medicine, vol. 174, no. 2, pp. 53-63, 2017.

[22] R. Li, W. Wang, and Z. Di, "Effects of human dynamics on epidemic spreading in Côte d'Ivoire," Physica A. Statistical Mechanics and its Applications, vol. 467, pp. 30-40, 2017.
[23] W. Liu, C. Liu, X. Liu, S. Cui, and X. Huang, "Modeling the spread of malware with the influence of heterogeneous immunization," Applied Mathematical Modelling. Simulation and Computation for Engineering and Environmental Systems, vol. 40, no. 4, pp. 3141-3152, 2016.

[24] P. Di Giamberardino and D. Iacoviello, "Optimal control of SIR epidemic model with state dependent switching cost index," Biomedical Signal Processing and Control, vol. 31, pp. 377-380, 2017.

[25] S. N. Langel, F. C. Paim, K. M. Lager, A. N. Vlasova, and L. J. Saif, "Lactogenic immunity and vaccines for porcine epidemic diarrhea virus (PEDV): Historical and current concepts," Virus Research, vol. 226, pp. 93-107, 2016.

[26] D. Greenhalgh, Y. Liang, and X. Mao, "Modelling the effect of telegraph noise in the SIRS epidemic model using Markovian switching," Physica A. Statistical Mechanics and its Applications, vol. 462, pp. 684-704, 2016.

[27] K. P. Hadeler, K. Dietz, and M. Safan, "Case fatality models for epidemics in growing populations," Mathematical Biosciences, vol. 281, pp. 120-127, 2016.

[28] W. Liu, C. Liu, Z. Yang, X. Liu, Y. Zhang, and Z. Wei, "Modeling the propagation of mobile malware on complex networks," Communications in Nonlinear Science and Numerical Simulation, vol. 37, pp. 249-264, 2016.

[29] S. Wen, J. Jiang, B. Liu, Y. Xiang, and W. Zhou, "Using epidemic betweenness to measure the influence of users in complex networks," Journal of Network and Computer Applications, vol. 78, pp. 288-299, 2017.

[30] C. Li, X. Yu, T. Huang, G. Chen, and X. He, "A generalized Hopfield network for nonsmooth constrained convex optimization: Lie derivative approach," IEEE Transactions on Neural Networks and Learning Systems, vol. 27, no. 2, pp. 308-321, 2016.

[31] M. Frasca and K. J. Sharkey, "Discrete-time moment closure models for epidemic spreading in populations of interacting individuals," Journal of Theoretical Biology, vol. 399, pp. 13-21, 2016.

[32] M. A. Andrews and C. T. Bauch, "The impacts of simultaneous disease intervention decisions on epidemic outcomes," Journal of Theoretical Biology, vol. 395, pp. 1-10, 2016.

[33] Y. Feng, L. Ding, Y.-H. Huang, and L. Zhang, "Epidemic spreading on weighted networks with adaptive topology based on infective information," Physica A. Statistical Mechanics and its Applications, vol. 463, pp. 493-502, 2016.

[34] G. Chen, C. J. Li, and Z. Y. Dong, "Parallel and Distributed Computation for Dynamical Economic Dispatch," IEEE Transactions on Smart Grid, vol. 8, no. 2, pp. 1026-1027, 2017.

[35] H. Li, G. Chen, T. Huang, and Z. Dong, "High-performance consensus control in networked systems with limited bandwidth communication and time-varying directed topologies," IEEE Transactions on Neural Networks and Learning Systems, pp. 1-12, 2016. 


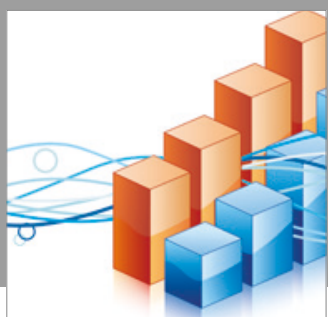

Advances in

Operations Research

vatersals

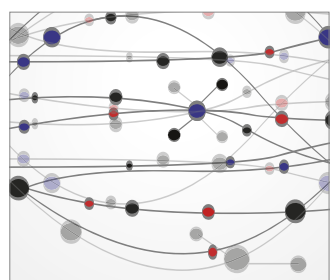

\section{The Scientific} World Journal
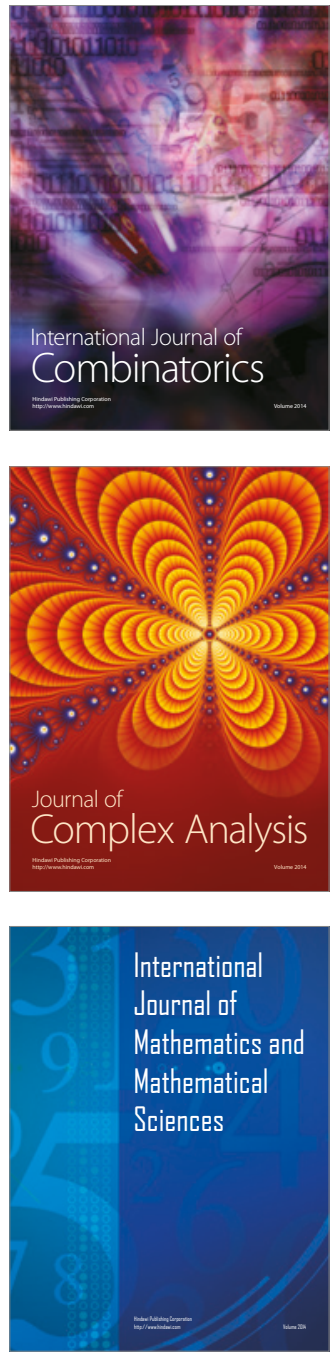
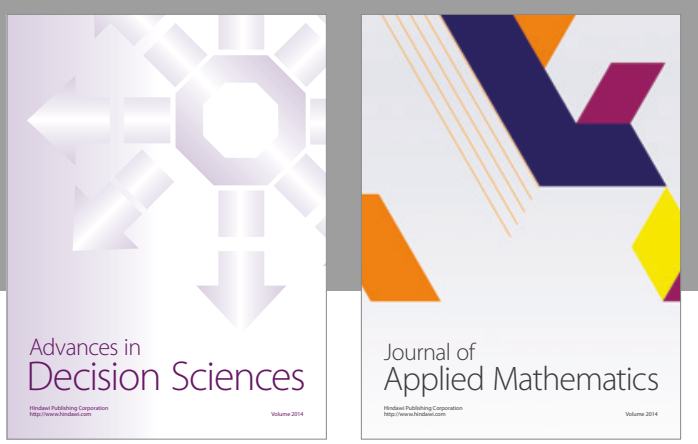

Algebra

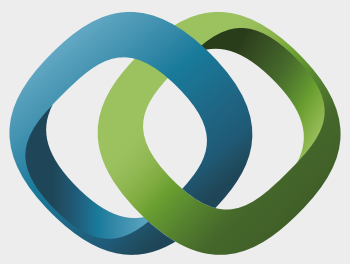

\section{Hindawi}

Submit your manuscripts at

https://www.hindawi.com
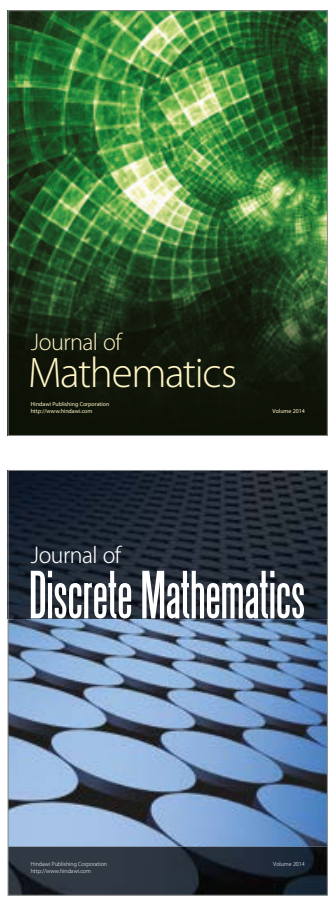

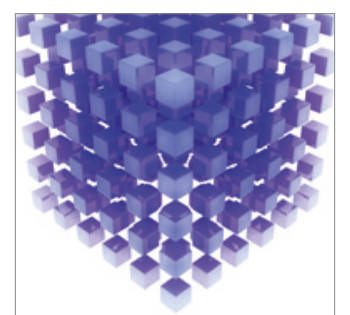

Mathematical Problems in Engineering
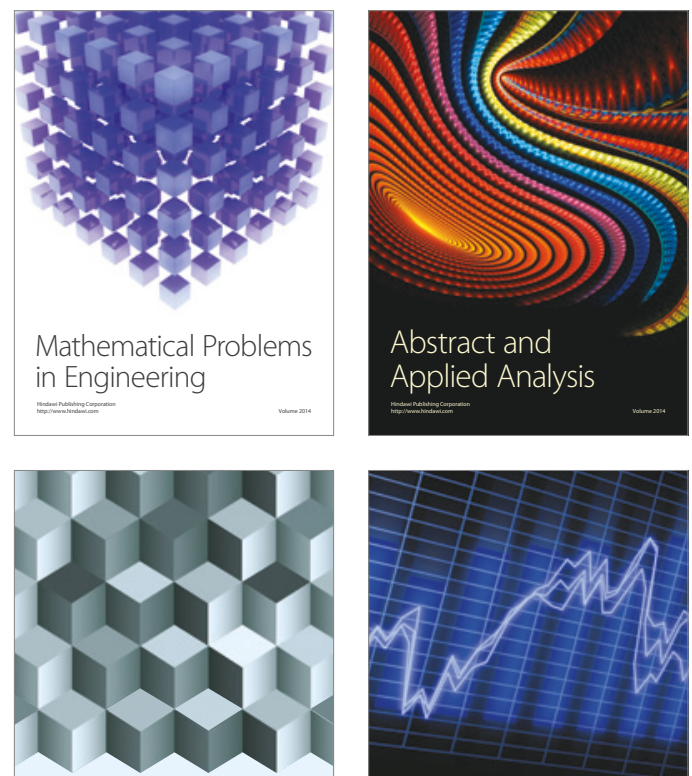

Journal of

Function Spaces

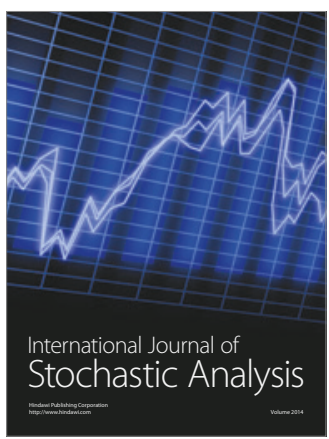

Probability and Statistics
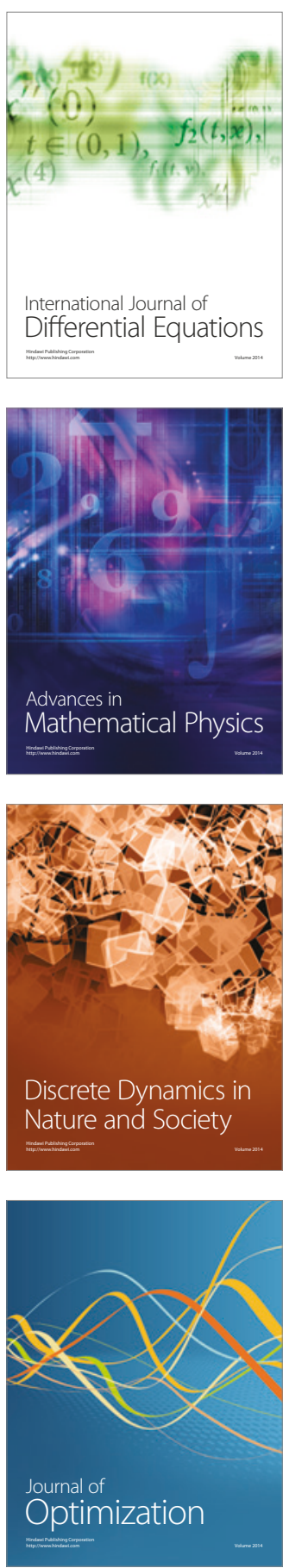